\title{
The electronic medical record and Patient-centered care
}

\author{
Neil J Nusbaum ${ }^{1}$ \\ ${ }^{1}$ Dept of Veteran Affairs, VA Central Western Massachusetts Health care System
}

\begin{abstract}
Background: One goal in EMR development should be to facilitate a patient-centered clinical encounter. Much prior EMR development has focused on capturing objective data, such as laboratory values and medication lists. Less attention has been devoted to the more complex task of capturing and analyzing data that incorporates the patient's concerns and preferences.
\end{abstract}

Methods: A literature search supplemented the author's own various experiences with one EMR (that used nationally by the Department of Veterans Affairs) from his various perspectives of a physician, an educator, and a Chief of Staff. This data was used to identify both opportunities and obstacles to promoting patient-centered care in an integrated care setting that relies heavily on an EMR. Qualitative analysis and suggestions are offered for how the EMR can individualize patient care, in support of a patient-centered approach.

Result: Three promising target areas in efforts to develop a patient-centered EMR are: elicitation of the chief complaint, conduct of health screening activities, and evaluation of health literacy. A range of strategies were identified, some of which may require information technology development, such as to facilitate patient direct entry of data into their own EMR.

Conclusion: EMR design can facilitate a more patient-centered clinical encounter. Beyond the benefits to the individual patient, patient-centric modifications to the EMR architecture may also facilitate quality improvement and research activities on patient centered care. In light of the widespread current discussions of a movement toward Accountable Care Organizations that use EMR, it will be especially important to ensure that the resulting care systems maintain a focus on the patient and not just on the system of care.

Key Words: Usability of Health Information, Health Promotion / Disease Prevention, Information Technology, Organization and Delivery of Care, Quality of Care 


\section{Introduction}

There has been considerable interest in making the clinical encounter more patient centered.[1] At the same time, in recent years there has been widespread exploration of electronic medical record (EMR) use in both inpatient and outpatient settings.[2] Clinicians wish to implement the EMR in such a way that it does not retard the emotional depth of the interaction between the physician and patient.[3] Accordingly, it is fruitful to consider efficient and harmonious ways in which the implementation of a patient-centered approach and introduction of EMR will proceed in concert.[ [ $\left.\begin{array}{llll}4 & 5 & 6 & 7\end{array}\right]$

The challenges in implementation of a patient-centered EMR differ somewhat according to the component of the patient record. The most rigidly structured components are the easiest to organize or capture into an electronic record, but the very rigidity of the structure may easily shift the center of focus to the electronic instrument and its computer interface.[8] The range of challenges and opportunities in creating a patient-centered EMR can be illustrated by considering three disparate components of the health care encounter: elicitation of the chief complaint, health screening activities, and evaluation of health literacy.

\section{The chief complaint}

Numerous observers have bemoaned the tendency of clinicians to quickly interrupt the patient's own description of their chief complaint at the initiation of a clinical encounter. The chief complaint is intended by its nature to represent a report by the patient of their subjective concern, and therefore, it does not need to correspond to any standard pathophysiological criteria of plausibility. The chief complaint classically represents a part of the history that is driven by the patient and not the physician. Among other purposes, a careful recording of the chief complaint can serve as the anchor for the rest of the patient encounter to help ensure that the patient's concerns are addressed. Fidelity of recording is particularly important where the history is gathered by one person such as a clinic nurse before the patient sees another individual such as the clinic doctor.

In order to make full use of the chief complaint it is desirable to build on emerging technology to allow the patient themselves to record their chief complaint in their own words rather than relying on the nurse or physician to serve as a scribe. In order to accomplish such direct patient entry, of course, the computer architecture must be structured to provide the capability for a patient to enter data into the EMR, whether from a computer keyboard, a tablet, or perhaps even by means of voice dictation software (such as could be used even by the visually impaired or those including children who lack literacy skills) some other device.[9] One then could allow the patient to electronically enter their chief complaint in response to the typical open-ended question of the main reason for their visit today. Their response could be imported verbatim into the patient record as the stated chief complaint.

In some cases, the clinician will find that the patient's chief complaint actually encapsulates well what the clinician perceives during the encounter to be the most 
important medical issue. In other cases, the chief complaint may represent a subjective concern of the patient alone, but one which the clinician regards as not a serious threat to health. In still other instances, the chief complaint may evidence a significant misunderstanding by the patient of the pathophysiology of their disease process, a topic on which the patient might benefit from focused patient education. A shared attentiveness to the patient's chief complaint needs to be maintained by the entire caregiving team throughout the course of the patient encounter.

In particular, it is important for the ongoing physician-patient relationship that the chief complaint has been appropriately addressed by the end of the encounter. A clear and legible verbatim transcript of the patient's chief complaint, such as one recorded digitally by the patient themselves before the encounter began, could well be integrated into the process of closing the patient encounter. This might be as straightforward as the clinician, toward the close of the encounter, paraphrasing the patient's chief complaint and then asking the patient themselves whether the patient felt that the chief complaint had been addressed to the patient's satisfaction. If the patient's answer is in the negative, the clinician (depending upon clinical needs and time constraints) could further address the chief complaint in closing the current visit and/or offer the patient a follow-up visit to address the chief complaint.

In general, having a legible verbatim version of the patient's chief complaint will facilitate its use not just for the current patient encounter but also as a reference point for future patient encounters. Although clinicians commonly elicit a chief complaint at the current visit, it is certainly less common for clinicians to search the record of chief complaints offered by the patient at prior visits. A history of repeated similar chief complaints on multiple prior visits may be a valuable relatively objective indicator of the chronicity of a medical problem, and so complement the patient's own present recollection of when the complaint first arose. A broad array of complaints over prior visits can similarly serve as a complement to the current elicitation of the review of systems, and may offer helpful clues to the presence of chronic multisystem disease.

\section{Use of the electronic medical record to target health screening}

A concern that regularly arises when the electronic medical record is introduced into a clinical setting is that the introduction of the electronic interface will, at least in the nearterm, reduce the efficiency of the interaction between physician and patient and thereby reduce the number of patients that can be seen during the workday. A poorly designed EMR can force the physician to spend time on computer- centered tasks such as screen navigation and data entry, at the expense of time for patient-centered tasks.

One way to alleviate this potential time barrier to introduction of the electronic medical record accordingly is to create early opportunities for the electronic medical record to increase the efficiency of the clinical interaction. Even a fairly rudimentary EMR can assist in creating efficiency in mechanical tasks, such as entering the date and time into progress notes. A bigger challenge is to structure the EMR so that it can support rather than impede the humanistic aspects of the physician-patient interaction. 
A concern of primary care physicians is that the time constraints of the clinical encounter leave relatively little time for patient counseling about health maintenance and health screening issues. Further exacerbating this constraint, bodies such as third-party payers may insist upon repeated counseling efforts upon a wide variety of topics. Scattered attention at a single visit to multiple counseling topics, if it creates the impression for the patient that all the counseling topics are of equal importance, also has the potential to dilute the impact of the interaction with regard to those topics of most significant concern to the individual patient's health.

An EMR menu could readily maintain a menu of pending health maintenance topics, offer both the physician and the patient an opportunity to prioritize which on the list should be addressed at the current visit, and to come to an agreement on steps (such as planning for an early follow-up visit) to address those items not resolved on the current visit. A lengthy and complex list of deferred reminders could prompt the EMR itself to suggest that the follow-up appointment be planned to allow sufficient length to accommodate the planned discussion agenda. The list of deferred items could be appended to the next clinic appointment as a popup screen in the EMR itself. The list might also be appended to any clinic reminders that are sent to the patient before the follow-up appointment.

One use of the medical record, which has already been translated into practice in some environments, is to target clinical interventions demographically. A simple example is to target clinician reminders for screening tests such as a mammogram [10] only for those patients for whom the respective test is appropriate according to their gender, age, and past medical history. Likewise clinical reminders in some EMR environments are automatically suppressed if they are not currently relevant to the specific patient, such as a patient who has already received their influenza immunization for that flu season.

Flu vaccine reminders in the EMR could be translated on the patient side into automated phone calls or mail or email to eligible patients, with the mode of communication employed chosen for the given patient in patient-centric fashion, in accordance with their personal preference as it has previously been recorded in their EMR. The EMR similarly can structure the encounter on the clinician side, so that an offer of flu vaccination can be made accordingly to clinician preference by the intake nurse, during the physician encounter, and/or at checkout after the physician encounter.

Screening interventions could further be targeted by use of patient electronic responses to questions regarding risk factors. Patients could respond to even very personal (or sensitive) questions using an electronic tool such as a PDA to record their answers[11], and indeed some patients may feel more comfortable responding to such questions on an electronic form[12] rather than verbally face-to-face with a clinician questioner. Answers to questions regarding issues such as recreational drug use or risky sexual behavior, for example, might influence whether a patient should be offered not only initial testing for HIV but also subsequent retesting if the initial test were negative.

The EMR may be a particularly useful device as well to share and simultaneously track 
share of patient care information across sites of care for the patient, such as across the entire structure of health care networks, while simultaneously tracking that sharing for HIPAA and similar regulatory purposes. Sharing of information in the EMR certainly should be an important component of the electronic integration of future accountable care organizations, if they are indeed to function as integrated entities. Efforts to develop the use of the EMR as a tool for patient centered care should embrace not just physicians and nurses, but also other clinical providers such as pharmacists. [13] Ideally, the EMR also should include and integrate not merely the data from face to face visits, but also patient concerns as expressed in electronic messages that may have been sent between visits. [14]

It should readily be possible to further increase the targeting of clinical reminders in the electronic medical record in a patient-specific fashion. Consider for example a patient with diabetes with comorbidities that is routinely seen in the office at three month intervals. If the patient has need for say four different topics to be raised (e.g. smoking cessation, depression screening, advice regarding exercise, advice regarding daily visual foot inspection) one could use the electronic medical record to sequence each of the topics to be raised one at a time over the course of a year at each quarterly visit rather than having all four topics scheduled to come up simultaneously at multiple times during the year.

The sequential approach would allow each of the topics in turn to receive adequate attention. If desired, one could also include a clinical reminder to the clinician of the topic that had been raised in counseling at the last prior visit so that it could be reinforced at the current visit. If the preceding visit had included a discussion of foot care, the clinician might well wish to briefly reinforce this topic at the current visit, and pay particular attention to the feet during a physical examination.

A still more sophisticated algorithm might include a prioritization of topics according to previous responses from the patient. If the patient is a smoker who has repeatedly rejected any consideration of smoking cessation then it might still be appropriate to raise the topic at least briefly at least once per year. On the other hand, if the patient had been in contemplation of smoking cessation on a recent visit, then the topic of smoking might appropriately be flagged for more detailed discussion at each of the succeeding four visits.

\section{Patient health literacy}

A collateral benefit of the approach described above is that it would enter in machinereadable format a sample of free text provided by the patient. The patient's text entry could readily be analyzed with tools such as those already bundled in a word processing program to analyze the level of complexity of the written passage. [15] The reading level of the patient text, or indeed even the patient's failure to provide any textual material at all in response to the request for it, might offer a ready clue to the clinician that the patient's literacy skills are limited.

A patient's lack of health literacy can be hidden from the clinician if the patient is purely 
a passive participant in their own health care. Encouraging the patient to ask questions has been embraced as a strategy to make the patient more active in their own care. An example is the "ask me three" questions approach: "Providers should always encourage their patients to understand the answers to: 1 . What is my main problem? 2. What do I need to do? 3. Why is it important for me to do this? ) [16]

The answer to the first of the three questions, "What is my main problem?" is the main problem as identified by the clinician by the close of the encounter. That answer by the clinician may or may not resemble the chief complaint, the main problem as seen by the patient at the start of the encounter. One strategy to help improve patient health literacy, therefore, may be to focus attention on how and why the clinician statement of the main problem differs from the patient statement of the chief complaint. The EMR could also be readily structured so that one task for the physician or checkout nurse is to review with the patient whether the patient has had those three "ask me three " questions each answered to the patient's satisfaction.

One of the challenges for a clinician as educator is to provide patient education whose form, content, and level of complexity are appropriate to the patient. Even for a given patient with a chronic condition, the education goals may evolve as the patient becomes more knowledgeable about that condition. [17] It might well be of interest for quality improvement or research purposes to track how the educational needs of the patient, as reflected in their chief complaint and/or in their "ask me three" questions, evolve over serial visits. It could similarly be instructive to observe from textual analysis of the chart whether the chart entries by the provider evolve in a corresponding fashion. Finally, as it may become more common for patients to read their own charts, it would be of interest to learn from patients if they felt the chart reflected their own understanding of what happened at the visit, if they found the chart itself difficult to read e.g. because of jargon, and indeed if such use of jargon at the actual visit had interfered with their understanding at the visit.

\section{Conclusions}

The EMR offers a powerful tool for implementing standardized approaches to care that may be very helpful in implementing clinical protocols and other interventions to decrease clinical omissions or other errors in a highly reproducible fashion. An EMR environment is particularly useful for addressing mechanical tasks, such as retrieving whether a patient has already had a flu shot this year. At the same time, however, the EMR has rich potential to support appropriate personalization of care. A sophisticated EMR can best meet the needs of an individual patient if it simultaneously supports care provision that is evidence based as well as care that is sensitive to the individual patient's emotional, educational and cultural context.[18] If a personal health record [19] is to be truly personal, it should incorporate concerns relevant to the particular patient. It is essential to develop the full potential of EMR implementation if the EMR is to actively support the paradigm of patient centered care.

Note: Personal opinions are those of the author, not official. 


\title{
Correspondence
}

\author{
Neil Jay Nusbaum \\ Dept. of Veteran Affairs \\ Email: NusbN@aol.com
}

\section{References}

[1] Brennan PF, Downs S, Casper G. Project HealthDesign: Rethinking the power and potential of personal health records. J Biomed Inform. 2010;43(5, Supplement 1):S3-S5. http://www.ncbi.nlm.nih.gov/pubmed/20937482

[2] Ludwick D, Doucette J. Adopting electronic medical records in primary care: lessons learned from health information systems implementation experience in seven countries. Int J Med Inform. Jan 2009;78(1):22-31. http://www.mece.ualberta.ca/ doucette/Publications/Ludwick-Doucette-IJMI-2009EMR.pdf

[3] Chan W, Stevenson M, McGlade K. Do general practitioners change how they use the computer during consultations with a significant psychological component? Int J Med Inform. Aug 2008;77(8):534-538. http://www.sciencedirect.com/science/article/pii/S138650560700175X

[4] Ventres W, Kooienga S, Marlin R. EHRs in the exam room: tips on patient-centered care. Fam Pract Manag. Mar 2006;13(3):45-47. http://www.aafp.org/fpm/2006/0300/p45.html

[5] Reid R, Wagner E. Strengthening primary care with better transfer of information. CMAJ. Nov 2008;179(10):987-988. http://www.cmaj.ca/content/179/10/987.full

[6] Detmer D, Bloomrosen M, Raymond, Tang P. Integrated personal health records: Transformative tools for consumer-centric care. BMC Medical Informatics and Decision Making 2008, 8:45. http://www.biomedcentral.com/1472-6947/8/45

[7] Murphy J. Patient as center of the health care universe: A closer look at patientcentered care. Nursing Economics 2011; 29(1):35-37.

[8] Rhodes P, Langdon M, Rowley E, Wright J, Small N. What does the use of a computerized checklist mean for patient-centered care? The example of a routine diabetes review. Qual Health Res. Mar 2006;16(3):353-376. http://www.ncbi.nlm.nih.gov/pubmed/16449686

[9] Moore BJ, Gaehde S, Curtis C. Architectural choices and challenges of integrating electronic patient questionnaires into the electronic medical record to support patientcentered care. AMIA Annu Symp Proc 2008; 2008:490-494. PMCID: PMC2655980 [10] Baron RJ. Quality improvement with an electronic health record: Achievable, but not automatic. Ann Int Med 2007;147:549-552.

http://www.annals.org/content/147/8/549.abstract [11] Bernabe-Ortiz A, Curioso WH, Gonzalez MA, Evangelista W, Castagnetto JM, Carcamo CP, et al. Handheld computers for self-administered sensitive data collection: A comparative study in Peru BMC Medical Informatics and Decision Making 2008, 8:11. http://www.biomedcentral.com/1472-6947/8/11 
[12] Ruland C, Starren J, Vatne T. Participatory design with children in the development of a support system for patient-centered care in pediatric oncology. $\mathrm{J}$ Biomed Inform. Aug 2008;41(4):624-635. http://www.sciencedirect.com/science/article/pii/S1532046407001116 [13] Frenzel JE. Using electronic medical records to teach patient-centered care. American J of Pharmaceutical Education 2010; 74(4) Article 71.

[14] Stiles RA, Deppen SA, Figaro MK, et al. Behind-the-scenes of patient-centered care: Content analysis of electronic messaging among primary care clinic providers and staff. Med Care. 2007;45(12):1205-1209. DOI: 10.1097/MLR.0b013e318148490c

[15] http://office.microsoft.com/en-us/word/HP051896011033.aspx . Website accessed August 7, 2011.

[16] http://www.npsf.org/askme3/. Website accessed August 7, 2011.

[17] Craft CE, Fowles JB, Kind AC, Kind EA, Richter SA. No change in physician dictation patterns when visit notes are made available online for patients. Mayo Clinic Proceedings 2011; 86(5):397

http://dx.doi.org/10.4065/mcp.2010.0785 [18] Snyder CF, Wu AW, Miller RS, Jensen RE, Bantug ET, Wolff AC. The Role of Informatics in Promoting Patient-Centered Care. Cancer J. 2011; 17(4):211-218. DOI: 10.1097/PPO.0b013e318225ff89

[19] Smith SP, Barefield AC. Patients meet technology: The newest in patient-centered care initiatives. Health Care Manag (Frederick). 2007;26(4):354-362.

PMID:17992110 